\title{
Large negative and positive delay of optical pulses in coherently prepared dense $R b$ vapor with buffer gas.
}

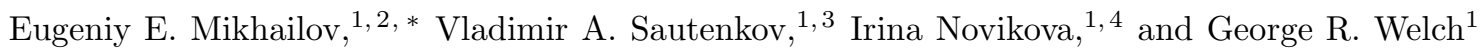 \\ 1 Department of Physics and Institute for Quantum Studies, \\ Texas AESM University, College Station, TX 77843 \\ ${ }^{2}$ Center for Space Research, Massachusetts Institute of Technology, Cambridge, MA 02139 \\ ${ }^{3}$ Lebedev Institute of Physics, 119991 Moscow, Russia \\ 4 Harvard-Smithsonian Center for Astrophysics, Cambridge, MA 02138
}

(Dated: August 23, 2018)

\begin{abstract}
We experimentally study the group time delay for a light pulse propagating through hot ${ }^{87} \mathrm{Rb}$ vapor in the presence of a strong coupling field in a $\Lambda$ configuration. We demonstrate that the ultraslow pulse propagation is transformed into superluminal propagation as the one-photon detuning of the light increases due to the change in the transmission resonance lineshape. Negative group velocity as low as $-c / 10^{6}=-80 \mathrm{~m} / \mathrm{s}$ is recorded. We also find that the advance time in the regime of the superluminal propagation grows linearly with increasing laser field power.
\end{abstract}

PACS numbers: 42.50. Gy, 42.50.-p, 42.25.Bs

\section{INTRODUCTION}

Coherent population trapping (CPT) occurs when three-level atoms interact with two coherent electromagnetic fields in a $\Lambda$ configuration. In this case, atoms are optically pumped into the non-interacting superposition of the two ground states (into the "dark" state), and the linear absorption of the optical fields is greatly reduced. In addition to the absorption cancellation, such atomic media exhibit steep nonlinear dispersion. This steep dispersion can result in significant reduction of the group velocity $v_{g}$ of a pulse propagating in a coherent medium, even though the refractive index $n$ is still very close to unity:

$$
v_{g}=\frac{c}{n+\omega \frac{\partial n}{\partial \omega}} .
$$

Group velocity as low as a few meters per second has been demonstrated experimentally in alkali atoms in BoseEinstein condensates [1], thermal atomic vapor [2, 3], ruby crystal at room temperature [4] and in Pr doped $\mathrm{Y}_{2} \mathrm{SiO}_{5}$ crystals [5]. See Ref. [6] for a comprehensive review of slow light-related research.

The amplitude and width of the transmission and dispersion resonances, and therefore the nonlinear properties of the medium, are largely determined by the time atoms stay in the dark state. In thermal alkali vapors this time is limited by the interaction time of the atoms with the laser fields, which in turn is usually limited by the average time-of-flight of an atom through the laser beam. To prolong the interaction time, an inert buffer gas is often added to the atomic vapor to slow the diffusion of the coherently prepared atoms through the laser beam [7, 8, 9]. However, it has been recently demon-

*Electronic address: evmik@leona.physics.tamu.edu strated that significant modification of the dark resonance is possible in atomic vapor in the presence of buffer gas [10, 11]. Namely, the initially symmetric narrow transmission resonance is transformed into an asymmetric dispersion-like lineshape and then into a (potentially narrower) nearly symmetric absorption resonance as the one-photon detuning of the laser fields from the excited state is increased. Therefore a far-detuned $\Lambda$ system may exhibit superluminal propagation of the probe pulse due to the steep anomalous dispersion associated with a narrow absorption line.

Although the superluminal propagation of a light pulse near resonant absorption or gain lines has been known and discussed for more than a century 12], interest in this effect has been revived recently by the observation of ultra-low negative group velocity 13, 14, 15, 16, 17, 18, 19, 20, 21]. Here we present an extensive study of the time delay of probe pulses propagating in a $\Lambda$ scheme under the conditions of CPT. We trace the modification of positive delay for zero one-photon detuning to negative delay for large one-photon detuning. Also we present the dependence of the delay time on total laser power.

\section{EXPERIMENTAL SETUP}

A schematic of the experimental setup is shown in Fig. 1b. An extended cavity diode laser is tuned to $5^{2} S_{1 / 2} F=2 \rightarrow 5^{2} P_{1 / 2} F^{\prime}=2$ transition of ${ }^{87} \mathrm{Rb}$ $(\lambda=795 \mathrm{~nm})$. The laser output is phase modulated using an electro-optic modulator (EOM) at a frequency close to the ${ }^{87} \mathrm{Rb}$ ground-state splitting $(6.835 \mathrm{GHz})$, so that one of the modulation sidebands is resonant with $5^{2} S_{1 / 2} F=1 \rightarrow 5^{2} P_{1 / 2} F=2$ transition, forming the $\Lambda$ system shown in Fig. 11 1 . The other sideband is fardetuned from all ${ }^{87} \mathrm{Rb}$ transitions, and has no effect on the phenomena described below. Approximately $7 \%$ of the total laser power is transferred to the probe field. After the EOM the probe and drive fields pass through 


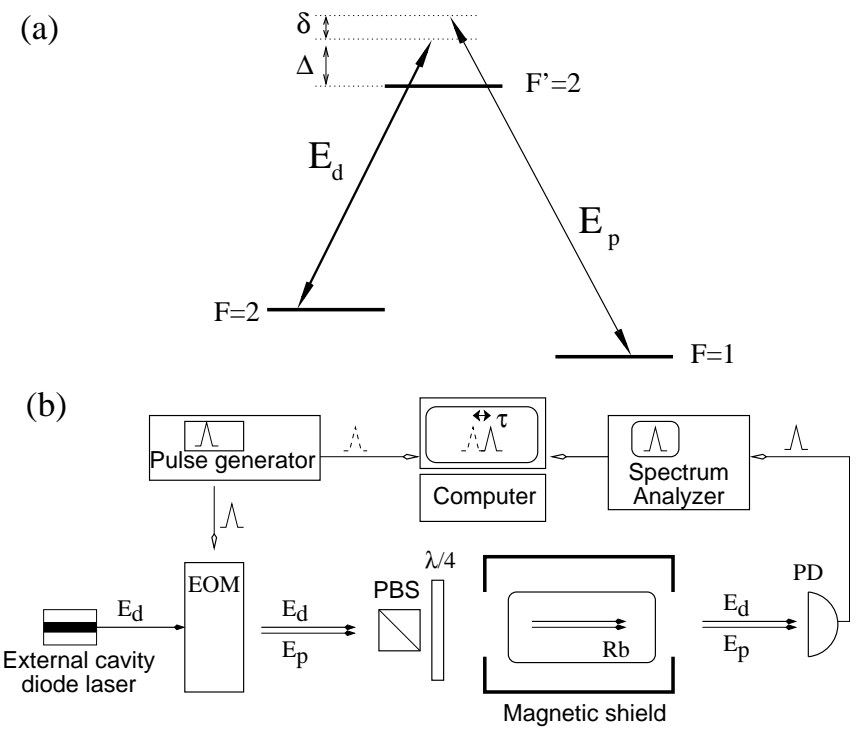

FIG. 1: (a) ${ }^{87} \mathrm{Rb}$ level diagram; (b) Schematic of the experimental apparatus.

a single mode optical fiber to ensure a Gaussian spatial intensity distribution, and their polarization is made circular by a high-quality polarizer followed by a quarter wave-plate. The overall light intensity is controlled by the rotation of an additional polarizer placed after the fiber. The diameter of the laser beam at the entrance of ${ }^{87} \mathrm{Rb}$ cell is approximately $7 \mathrm{~mm}$.

The experiment is conducted using a $2.5 \mathrm{~cm}$ long cylindrical glass cell filled with a mixture of isotopically enhanced ${ }^{87} \mathrm{Rb}$ and 30 Torr of Ne buffer gas. The cell is placed inside a 3 layer magnetic shield to screen out the Earth's magnetic field, and maintained at $68^{\circ} \mathrm{C}$, which results in an atomic density of ${ }^{87} \mathrm{Rb}$ vapor $N=$ $4.7 \times 10^{11} \mathrm{~cm}^{-3}$. After the cell the optical fields are mixed with an additional optical field shifted by $60 \mathrm{MHz}$ with respect to the original laser frequency, and the amplitude of the beat signal between this field and and the probe field is used to monitor the changes in the transmitted probe field intensity.

\section{EXPERIMENTAL RESULTS}

To observe the modification of the dark resonance lineshape we change the one-photon detuning $\Delta$ by changing the laser frequency, and then record the probe field transmission as a function of two-photon detuning by scanning the microwave frequency driving the EOM. In the case of a nearly resonant $\Lambda$ system $(\Delta=0)$, a narrow transmission resonance is observed due to coherent population trapping. An example of such a dark resonance is shown in Fig. 27. As the one-photon detuning increases, the shape of the resonance changes, and for large detuning it becomes almost purely absorptive (see Fig. 2b). Note that the widths of both resonances are

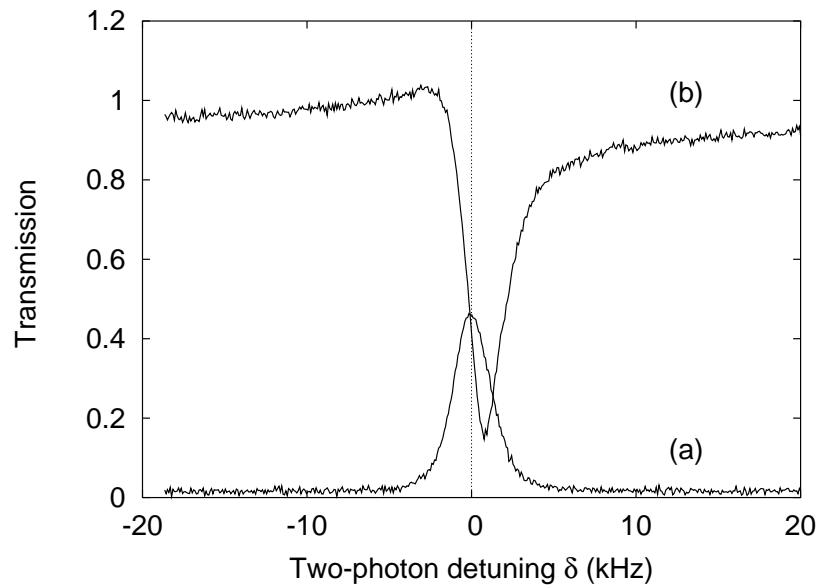

FIG. 2: The probe field transmission resonance for (a) zero one-photon detuning $\Delta=0$ and (b) for $\Delta=1.45 \mathrm{GHz}$. The output signals are shown on the same scale and normalized to the non-absorbed transmission. The total power of the laser beam is $400 \mu \mathrm{W}$.

similar $(\approx 2.5 \mathrm{kHz})$ and are determined by the decay rate of the ground-state atomic coherence.

The corresponding changes in the atomic dispersion from normal $(\partial n / \partial \omega>0)$ to anomalous $(\partial n / \partial \omega<0)$ produces a modification of the pulse delay from positive (slow light) to negative (superluminal regime, or fast light). Examples of such pulses are shown in Fig. 3 As expected the transmitted pulse for the resonant probe field is delayed with respect to the reference for $\tau=$ $370 \mu \mathrm{s}$, whereas for the far-detuned $\Lambda$ system the maximum of the output pulse leaves the cell noticeably earlier than the input. For the laser detuning of $\Delta=1.45 \mathrm{GHz}$ the advance time is $300 \mu \mathrm{s}$, which corresponds to a group index $n_{g} \simeq-4 \times 10^{6}$.

It is easy to see that only minimal reshaping of both retarded and advanced probe pulses is observed. In either case, the output pulses are still very close to the original Gaussian waveform, although their widths are slightly reduced. For a $1 \mathrm{~ms}$ duration input probe pulse the duration of the transmitted pulse is $0.94 \mathrm{~ms}$ in the case of slow light propagation and $0.81 \mathrm{~ms}$ in the case of superluminal propagation.

It is interesting to track the variation of the group velocity as the shape of the dark resonance changes from transmission to absorption with increasing laser detuning. This dependence of the delay time on the laser frequency is shown in Fig. [4. This dependence is measured in the following way: for each one photon detuning we adjust the the two-photon tuning of the probe field to the transmission peak (maximum or minimum) by adjusting the EOM modulation frequency. Then we propagate a Gaussian temporal probe field pulse through the medium and measure its relative delay with respect to the reference pulse. Close to atomic resonance $(\Delta<1 \mathrm{GHz})$ the EIT transmission peak is observed, although it becomes asymmetric as the laser detuning increases. The gap in 


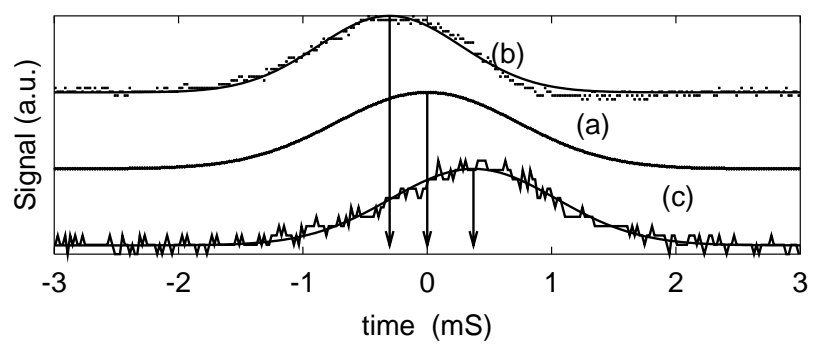

FIG. 3: Examples of transmitted pulses: (a) reference pulse (no atoms); (b) advanced pulse recorded at one-photon detuning $\Delta=1.45 \mathrm{GHz}$ (total laser power was $700 \mu \mathrm{W}$ ); (c) retarded pulse recorded at $\Delta=0$ (total laser power $145 \mu \mathrm{W}$ ). Solid lines represents the Gaussian lineshape. The amplitudes of all pulses are normalized for easier comparison.

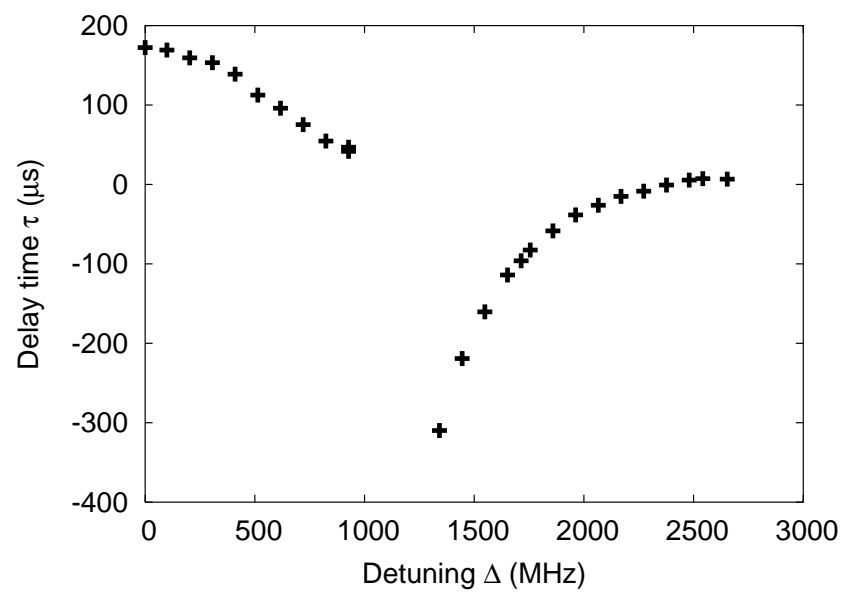

FIG. 4: Dependence of the positive and negative pulse delays on one photon laser detuning. Total power of the laser beam is $400 \mu \mathrm{W}$.

measurements between $1 \mathrm{GHz}$ and $1.4 \mathrm{GHz}$ corresponds to the range where the dark resonance lineshape is too dispersion-like to find a meaningful peak. Although theoretically both slow and superluminal group velocities might be measured in this case for two slightly different two-photon detunings, in practice it is rather difficult to perform such measurements. In this regime the measured group velocity is extremely sensitive to any changes in the one-photon detuning, and therefore the instability of the laser frequency creates a huge variation of the measured pulse delays. Once the absorption resonance begins to dominate (for $\Delta>1.4 \mathrm{GHz}$ ), we follow the minimum of the probe transmission by again adjusting the twophoton detuning appropriately.

We have also studied the dependence of the group velocity on the drive field power. We measure the pulse delay both for near-resonant (EIT) and far-detuned fields (enhanced absorption resonance) as the laser intensity is changed. The results are shown in Fig. 2] One can see that in the case of slow pulse propagation the group delay is inversely proportional to the laser intensity. This

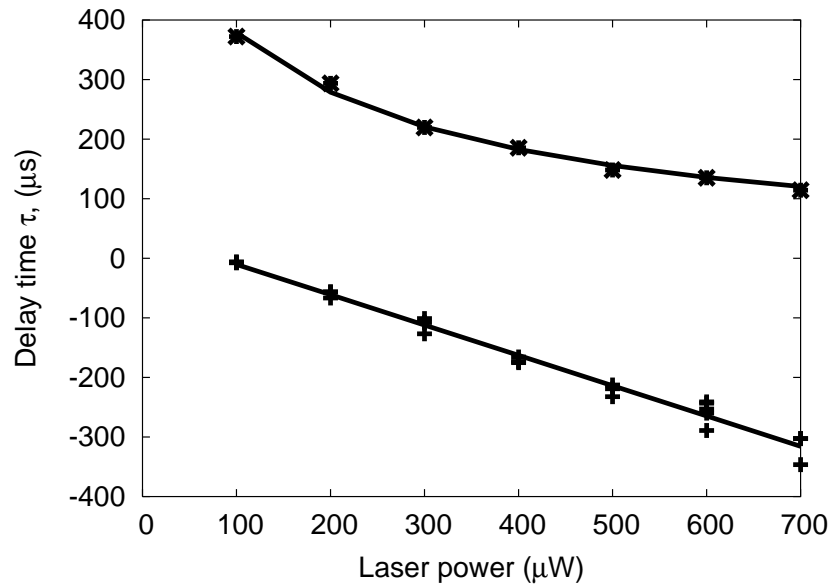

FIG. 5: The dependence of the positive and negative pulse delays on the laser power. The two graphs are for $\Delta=0$ (x) and $\Delta=1.44 \mathrm{GHz}(+)$. Solid lines represent fits to the experimental data for the two cases: if the lasers are tuned to the atomic resonances, $\tau \propto 1 /|\Omega|^{2}$, and for the far-detuned $\Lambda$ system $\tau \propto-|\Omega|^{2}$.

dependence is well described by the power broadening of the dark resonance [2]:

$$
\tau \simeq \frac{3}{8 \pi} N \lambda^{2} L \frac{\gamma_{r}}{|\Omega|^{2}} .
$$

This assumes the usual EIT conditions, namely $|\Omega|^{2} \gg$ $\gamma_{0} \gamma$ and $|\Omega|^{2} \gg \sqrt{\gamma_{0} / \gamma} W_{D}$, where $\Omega$ is the drive field Rabi frequency, $\gamma_{r}$ and $\gamma$ are the radiative and total decay rates of the excited states, $W_{D}$ is the width of the Doppler-broadened absorption line, and $\gamma_{0}$ is the dark state decoherence rate.

Very different behavior is observed for the superluminal pulse propagation. Namely, the advance time linearly increases with the laser power, which means that the steepness of the anomalous dispersion increases as well. This behavior may be explained by the fact that for the far-detuned $\Lambda$ system the width of the absorption resonance is much less affected by power broadening. The asymptotic behavior may be deduced from the general expression for the dark resonance width [1]

$$
\gamma_{\mathrm{EIT}} \simeq \gamma_{0}+\frac{\gamma^{2}|\Omega|^{4}}{2 \gamma_{0} \Delta^{4}}
$$

The experimental measurements of the resonance width vs. laser power are shown in Fig. [6] and are in good agreement with this expression. At the same time, the amplitude of the resonance is directly proportional to the laser power. The combination of these two effects provides the linear growth of the atomic dispersion and the probe pulse advance time.

We can check this conclusion in the following way. The nonlinear dispersion of the atomic medium may be calculated analytically using the well-known density matrix equations for a three-level $\Lambda$ system, if the motion of the 


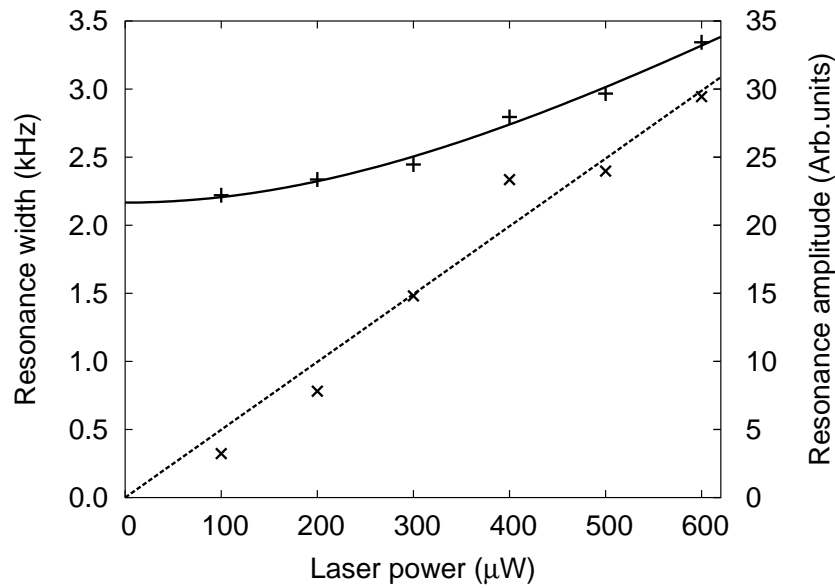

FIG. 6: The width $(+)$ and the amplitude (x) of the absorption resonance as a function of the laser power. The data are taken for one photon detuning $\Delta=1.44 \mathrm{GHz}$.

atoms is neglected. Following the calculations presented in 11] we find the time delay is given by:

$$
\begin{aligned}
\tau= & \frac{3}{8 \pi} N \lambda^{2} L \gamma_{r} \frac{\gamma^{2}+\Delta^{2}}{2 \gamma_{0} \Delta^{2}+\gamma|\Omega|^{2}} \times \\
& \frac{\gamma|\Omega|^{4}-2|\Omega|^{2} \frac{\Delta^{2}}{\gamma^{2}+\Delta^{2}}\left(\gamma_{0} \Delta^{2}+\gamma|\Omega|^{2}\right)}{\gamma_{0}^{2} \Delta^{2}\left(\gamma^{2}+\Delta^{2}\right)+\gamma^{2}|\Omega|^{4}}
\end{aligned}
$$

Although this expression is very cumbersome it reduces to Eq. (2) at one-photon resonance $(\Delta=0)$. A simple asymptotic behavior of the time delay may be also found for large detunings $\Delta^{2} \gg \gamma / \gamma_{0}|\Omega|^{2}$ we find

$$
\tau \simeq-\frac{3}{8 \pi} N \lambda^{2} L \frac{\gamma_{r}|\Omega|^{2}}{\gamma_{0}^{2} \Delta^{2}} .
$$

This linear increase in pulse advance time with laser power is just what is seen in Fig. 5

\section{DISCUSSION}

As previously mentioned, both large positive and negative group delay are the manifestation of steep atomic dispersion, either normal or anomalous. This means that the accumulated phase of an electromagnetic field traversing such a medium is very sensitive to even small variations in its frequency. Therefore, it may be measured with high precision if phase-sensitive measurements of the output field are made. The cancellation of the linear absorption is undoubtedly the biggest advantage of the steep dispersion associated with EIT, and has been widely exploited for precision magnetic field measurements $8,222,23,24,25,[26]$ and atomic standards 27, 28, 29, 30].
In this light, our experimental data suggest that the narrow absorption resonances observed in buffered atomic cells in a far-detuned $\Lambda$ system are also good candidates for precision measurements. We can estimate the phase $\Delta \varphi$ acquired by the probe field in this regime as:

$$
\Delta \varphi \approx \frac{\omega}{c} L \frac{\partial n}{\partial \omega} \delta \approx \tau \gamma_{\mathrm{EIT}}
$$

where we assume that the detuning of the probe field from the center of the absorption resonance $\delta$ is on the order of the resonance width $\gamma_{\text {EIT }}$. Substituting the experimental value for the group advance time $\tau \simeq 0.3 \mathrm{~ms}$ and for the resonance width $\gamma \simeq 2.5 \mathrm{kHz}$, we find the maximum phase difference to be about 5 rad, which is on the same order of magnitude as the large polarization rotation angle in nonlinear Faraday rotation 31] and the large phase shifts induced by gradient magnetic fields in stored light experiments 32 ].

\section{CONCLUSION}

We have studied the propagation of a weak probe pulse in an atomic cell filled with optically thick ${ }^{87} \mathrm{Rb}$ vapor and Ne buffer gas for various one-photon detunings from the upper atomic state of the $\Lambda$ system. The pulse propagation undergoes a transition from an ultra-slow to a superluminal regime as the dark resonance lineshape changes from a narrow transmission to a narrow absorption resonance. In the latter case, advance time as long as $300 \mu$ s has been observed, which is equivalent to a negative group velocity of $v_{g} \simeq-80 \mathrm{~m} / \mathrm{s}$.

We have also studied the dependence of the group delay on the laser power. While the group velocity under EIT conditions increases linearly, in agreement with the theoretical predictions, we see a linear growth of negative group delay with the laser power. The main reason is that for large one-photon detuning the contrast of the absorption resonance increases with laser intensity, whereas the change in the resonance width is negligible.

The large anomalous dispersion associated with the reported absorption resonances is associated with high sensitivity of the phase of the probe field to the frequency variations, which may be find application in precision measurements.

\section{Acknowledgments}

The authors thank Yuri V. Rostovtsev, Andrey B. Matsko, A. Zhang and M. O. Scully for useful discussions. This work was supported by the the Office of Naval Research, AFOSR, and the DARPA. 
[1] L. V. Hau, S. E. Harris, Z. Dutton, and C. H. Behroozi, Nature 397, 594 (1999).

[2] M. M. Kash, V. A. Sautenkov, A. S. Zibrov, L. Hollberg, G. R. Welch, M. D. Lukin, Y. Rostovtsev, E. S. Fry, and M. O. Scully, Phys. Rev. Lett. 82, 5229 (1999).

[3] D. Budker, D. F. Kimball, S. M. Rochester, and V. V. Yashchuk, Phys. Rev. Lett. 83, 1767 (1999).

[4] M. Bigelow, N. Lepeshkin, and R. Boyd, Phys. Rev. Lett. 90, 113903 (2003).

[5] A. V. Turukhin, V. S. Sudarshanam, M. S. Shahriar, J. A. Musser, B. S. Ham, and P. R. Hemmer, Phys. Rev. Lett. 88, 023602 (2002).

[6] A. B. Matsko, O. Kocharovskaya, Y. Rostovtsev, G. R. Welch, A. S. Zibrov, and M. O. Scully, Advan Atom Mol Opt Phys 46, 191 (2001).

[7] S. Brandt, A. Nagel, R. Wynands, and D. Meschede, Phys. Rev. A 56, R1063 (1997).

[8] R. Wynands and A. Nagel, Appl. Phys. B 68, 1 (1998).

[9] M. Erhard and H. Helm, Phys. Rev. A 63, 043813 (2001).

[10] E. E. Mikhailov, V. A. Sautenkov, Y. V. Rostovtsev, and G. R. Welch, LANL e-Print archive (2003), URL http://arxiv.org/abs/quant-ph/0309151

[11] E. E. Mikhailov, I. Novikova, Y. V. Rostovtsev, and G. R. Welch, LANL e-Print archive (2003), URL http://arxiv.org/abs/quant-ph/0309171

[12] R. Y. Chiao, Amazing Light (Springer, New York, 1996).

[13] L. J. Wang, A. Kuzmich, and A. Dogariu, Nature 406, 277 (2000).

[14] A. Dogariu, A. Kuzmich, and L. J. Wang, Phys. Rev. A 63, 053806 (2001).

[15] A. Kuzmich, A. Dogariu, L. J. Wang, P. W. Milonni, and R. Y. Chiao, Phys. Rev. Lett. 86, 3925 (2001).

[16] A. Godone, F. Levi, and S. Micalizio, Phys. Rev. A 66, 043804 (2002).

[17] A. M. Akulshin, A. Cimmino, A. I. Sidorov, P. Han- naford, and G. I. Opat, Phys. Rev. A 67, 011801(R) (2003).

[18] A. M. Akulshin, A. Cimmino, A. I. Sidorov, R. McLean, and P. Hannaford, J. Opt. B: Quantum Semiclass. Opt. 5, S479 (2003).

[19] M. Bigelow, N. Lepeshkin, and R. Boyd, Science 301, 200 (2003).

[20] M. Stenner, D. Gauthier, and M. Neifeld, Nature 425, 695 (2003).

[21] K. Kim, H. Moon, C. Lee, S. Kim, and J. Kim, Phys. Rev. A 68, 013810 (2003).

[22] M. O. Scully and M. Fleischhauer, Phys. Rev. Lett. 69, 1360 (1992).

[23] M. Fleischhauer and M. O. Scully, Phys. Rev. A 49, 1973 (1994).

[24] M. Stahler, S. Knappe, C. Affolderbach, W. Kemp, and R. Wynands, Europhysics Lett. 54, 323 (2001).

[25] D. Budker, D. F. Kimball, S. M. Rochester, V. V. Yashchuk, and M. Zolotorev, Phys. Rev. A 62, 043403 (2000).

[26] I. Novikova and G. R. Welch, J. Mod. Opt. 49, 349 (2002).

[27] J. Vanier, A. Godone, and F. Levi, Phys. Rev. A 58, 2345 (1998).

[28] J. Kitching, S. Knappe, and L. Hollberg, Appl. Phys. Lett. 81, 553 (2002).

[29] M. Merimaa, T. Lindvall, I. Tittonen, and E. Ikonen, J. Opt. Soc. Am. B 20, 273 (2003).

[30] J. Vanier, M. W. Levine, D. Janssen, and M. J. Delaney, IEEE Trans. Instrum. Meas. 52, 822 (2003).

[31] I. Novikova, A. B. Matsko, and G. R. Welch, Opt. Lett. 26, 1016 (2001).

[32] A. Mair, J. Hager, D. F. Phillips, R. L. Walsworth, and M. D. Lukin, Phys. Rev. A 65, 031802 (2002). 